\title{
General null Lagrangians, exact gauge functions and forces in Newtonian mechanics
}

\author{
Z. E. Musielak and T. B Watson \\ Department of Physics, University of Texas at Arlington, Arlington, TX 76019, USA
}

\begin{abstract}
A method to construct general null Lagrangians and their exact gauge functions is developed. The functions are used to define classical forces independently from Newtonian dynamics. It is shown that the forces generated by the exact gauge functions allow for the conversion of the first Newton equation into the second one. The presented approach gives new insights into the origin of forces in Classical Mechanics.
\end{abstract}

\section{Introduction}

In Newtonian mechanics, the three laws of dynamics are independent, and the second law, $F=m a$, is used to define the force $F$ [1]. Moreover, the second law is also used to define the inertial mass $m$, which is the proportionality factor between the force $F$ and the acceleration $a$ produced by it [2]. Hence, "the unknown is defined by the unknown" [1]. A possible solution of this circular definition problem is proposed in this Letter, where a novel method to introduce forces to Newtonian dynamics is presented. The method is also used to demonstrate that the Newton law of inertia can be converted into the second Newton law of dynamics, which removes their independence.

The presented method uses gauge functions that are similar to those that appear when the Galilean invariance of Newton's law of inertia is established [3]. The invariance requires the equation to preserve its form when the transformations representing the Galilean group of the metric are performed [4]. Thus, the Galilean transformations introduce the Galilean gauge that makes the equation invariant but not its corresponding Lagrangian [3,5]. Recently, the gauge functions were constructed for the Newtonian law of inertia and used to make the Lagrangian Galilean invariant [6].

In this Letter, the previous results [6] are generalized by introducing the exact gauge functions that require additional conditions to be imposed on the action [7]. Using these functions, the corresponding null Lagrangians are derived $[8,9,10]$ and the Galilean invariance of both the Newton law of inertia and its Lagrangian is established. The main advantage of this generalization is that it allows for the introduction of a general time-dependent force to the first Newton equation, thus converting this equation into the second Newton equation and implicitly preserving its Galilean invariance. This is our main result that gives new insights into the origin of forces in Classical Mechanics (CM), and in addition solves 
the longstanding problem of circular definitions of forces and inertial masses in Newtonian dynamics $[1,2]$.

\section{Galilean gauge function}

The metric of Galilean space and time is invariant with respect to all transformations that form the Galilean group of the metric $[4,5]$. Let $(x, y, z)$ be Cartesian coordinates associated with inertial frames of reference that move with respect to each other with constant velocity $v_{o}$, and let $t$ be time that is the same in all these frames. According to Newton's law of inertia, motion of a classical body is rectilinear and uniform with respect to any inertial frame.

In one-dimension (along $x$ ) the law of inertia is then given by

$$
\hat{D} x(t) \equiv \frac{d^{2} x(t)}{d t^{2}}=\ddot{x}(t)=0,
$$

where $x(t)$ is a dynamical variable of the body in one inertial frame. The Lagrangian for this equation can be written in its standard form as

$$
L_{s}[\dot{x}(t)]=\frac{1}{2} c_{o}[\dot{x}(t)]^{2},
$$

where $c_{o}$ is an arbitrary constant that represents inertial mass in CM.

Let $(x, t)$ and $\left(x^{\prime}, t^{\prime}\right)$ be inertial frames moving with respect to each other with the velocity $v_{o}=$ const, and let the system's origins be the same at $t=t^{\prime}=t_{o}=o$. Then the Galilean transformations between these two frames are: $x^{\prime}=x-v_{o} t$ and $t^{\prime}=t$. Let the initial conditions be $\left.u(t)\right|_{t=0}=\left.\dot{x}(t)\right|_{t=0}=u_{0}$ and $\left.x(t)\right|_{t=0}=x_{o}$, so that the solution of Eq. (1) can be written as $x(t)=u_{o} t+x_{o}$ and $x^{\prime}(t)=\left(u_{o}-v_{o}\right) t+x_{o}$, where $x_{o}=x_{o}^{\prime}$.

Applying these Galilean transformations to Eqs (11) and (2), we find that $\hat{D} x(t)=0$ and $\hat{D} x^{\prime}(t)=0$ are of the same form, thus, Galilean invariant. However, $L_{s}[\dot{x}(t)]=L_{s}^{\prime}\left[\dot{x}^{\prime}(t)\right]+$ $L_{G s}^{\prime}\left[x^{\prime}(t)\right]$, where

$$
L_{G s}^{\prime}\left[\dot{x}^{\prime}(t)\right]=c_{o}\left[\dot{x}^{\prime}(t)+\frac{1}{2} v_{o}\right] v_{o} .
$$

It is easy to verify that $L_{G s}^{\prime}\left[\dot{x}^{\prime}(t)\right]$ is the null Lagrangian [2] and that its Galilean gauge function [4] is

$$
\Phi_{G s}^{\prime}\left[x^{\prime}(t), t\right]=c_{o}\left[x^{\prime}(t)+\frac{1}{2} v_{o} t\right] v_{o} .
$$

The fact that the Galilean gauge function can be removed and $L_{s}[\dot{x}(t)]$ can be made Galilean invariant was recently shown [6] using other gauge functions. In the following, those previous results are generalized by introducing the exact gauge functions, which are later used to define forces in Newton's equations. 


\section{Exact gauge function}

The gauge function previously constructed [6] to make the standard Lagrangian for the law of inertia to be Galilean invariant can be written as

$$
\Phi[x(t), t]=\frac{1}{2} c_{1} x^{2}(t)+c_{2} x(t) t+c_{4} x+c_{6} t .
$$

where $c_{1}, c_{2}, c_{4}$ and $c_{6}$ are constants to be determined. After the Galilean transformations of $\Phi[x(t), t]$, the resulting $\Phi^{\prime}\left[x^{\prime}(t), t\right]$ is of the same form as Eq. (5) and the remaining terms are used to remove $\Phi_{G s}^{\prime}\left[x^{\prime}(t)\right]$ given by Eq. (41). This guarantees that the Lagrangians $L_{s}[\dot{x}(t)]$ and $L_{G s}[\dot{x}(t)]$ are of the same form [6]. In the following, the same procedure is used but for the newly defined exact gauge function, which also allows introducing forces into Newtonian dynamics.

We generalize $\Phi[x(t), t]$ by replacing the constants in Eq. (5) by functions of the independent variable that are continuous and at least twice differentiable, and obtain

$$
\Phi_{n}[x(t), t]=\frac{1}{2} f_{1}(t) x^{2}(t)+f_{2}(t) x(t) t+f_{4}(t) x+f_{6}(t) t
$$

where $f_{1}(t), f_{2}(t), f_{4}(t)$ and $f_{6}(t)$ are to be determined. Note that each term with a different function $f(t)$ is a partial gauge function.

Since the total derivative of $\Phi_{n}[x(t), t]$ gives the null Lagrangian $L_{n}[\dot{x}(t), x(t), t]$, we may write the action as

$$
\begin{aligned}
A\left[x(t) ; t_{e}, t_{o}\right]= & \int_{t_{o}}^{t_{e}}\left(L_{s}+L_{n}\right) d t=\int_{t_{o}}^{t_{e}} L_{s} d t+\int_{t_{o}}^{t_{e}}\left[\frac{d \Phi_{n}}{d t}\right] d t \\
& =\int_{t_{o}}^{t_{e}} L_{s} d t+\left[\Phi_{n}\left(t_{e}\right)-\Phi_{n}\left(t_{o}\right)\right],
\end{aligned}
$$

where $t_{o}$ and $t_{e}$ denote the initial and final times. The gauge functions at the end points are constants, so they do not conflict with Hamilton's Principle that requires $\delta A[x(t)]=0$ but they do add the difference between these constants to the value of the action. In other words, the value of the action is affected by the values of the gauge functions at the end points.

Let $\Phi_{n}[x(t), t]$ be the exact gauge function if, and only if, the value of the action remains unchanged, which means that both constants are either zero or the difference between them is zero; following [7], we assume the former condition and use it to determine the end values of the arbitrary functions in the exact gauge function. Other conditions on these functions are imposed by the Galilean invariance, which is now considered.

\section{Galilean invariance}

To establish Galilean invariance of the law of inertia (see Eq. 1) and its standard Lagrangian (see Eq. 2), we perform the Galilean transformations of $\Phi_{n}[x(t), t]$ and obtain 
$\Phi_{n}^{\prime}\left[x^{\prime}(t), t\right]$, and the following Galilean gauge function

$$
\Phi_{G n}^{\prime}\left[x^{\prime}(t)\right]=f_{1}(t)\left[x^{\prime}(t)+\frac{1}{2} v_{o} t\right] v_{o} t+\left[f_{2}(t)+f_{4}(t)\right] v_{o} t .
$$

To make the Lagrangian $L_{s}[\dot{x}(t)]$ Galilean invariant, it is required that the total derivative of the Galilean gauge (see Eq. 4) and the Galilean gauge function is zero, which means that the extra terms resulting from the Galilean transformations are eliminated. Thus, the condition for the Galilean invariance can be written as

$$
\frac{d}{d t}\left(\Phi_{G s}^{\prime}\left[x^{\prime}(t)\right]+\Phi_{G n}^{\prime}\left[x^{\prime}(t)\right]\right)=0
$$

or $\Phi_{G s}^{\prime}\left[x^{\prime}(t)\right]+\Phi_{G n}^{\prime}\left[x^{\prime}(t)\right]=\mathrm{c}=$ const. Using $x^{\prime}(t)=\left(u_{o}-v_{o}\right) t+x_{o}$, we obtain

$$
\begin{gathered}
f_{2}(t)=f_{1}(t)\left(\frac{1}{2} v_{o}-u_{o}\right), \\
f_{4}(t)=c_{o}\left(\frac{1}{2} v_{o}-u_{o}\right)-f_{1}(t) x_{o},
\end{gathered}
$$

and $c=c_{o} v_{o} x_{o}$.

Then, the exact gauge function is given by

$$
\Phi_{n}[x(t), t]=\frac{1}{2}\left[v_{o} t-x(t)\right] f_{1}(t) x(t)+c_{o}\left(\frac{1}{2} v_{o}-u_{o}\right) x(t)+f_{6}(t) t .
$$

Taking $t_{o}=0$, we determine the conditions required that the functions $f_{1}(t)$ and $f_{6}(t)$ satisfy in order for $\Phi_{n}[x(t), t]$ to be is exact. These conditions are

$$
f_{1}(0)=\frac{2 c_{o}}{x_{o}}\left(\frac{1}{2} v_{o}-u_{o}\right)
$$

and

$$
f_{1}\left(t_{e}\right)=\frac{2 u_{o}-v_{o}-2 t_{e} f_{6}(t)}{v_{o} t_{e}-x_{e}}
$$

shows that the end value of $f_{1}\left(t_{e}\right)$ depends also on $f_{6}\left(t_{e}\right)$, which remains undetermined.

Having obtained the exact gauge function $\Phi_{n}[x(t), t]$, we find the following exact null Lagrangian

$$
\begin{aligned}
L_{n}[\dot{x}(t), x(t), t]=\frac{1}{2} v_{o} & {[\dot{x}(t) t+x(t)] f_{1}(t)-f_{1}(t) \dot{x}(t) x(t)+\frac{1}{2}\left[v_{o} t-x(t)\right] \dot{f}_{1}(t) x(t) } \\
+ & \frac{1}{2}\left[v_{o}-2 u_{o}\right] c_{o} \dot{x}(t)+\left[\dot{f}_{6}(t) t+f_{6}(t)\right] .
\end{aligned}
$$

This null Lagrangian makes the standard Lagrangian given by Eq. (2) the Galilean invariant, which means that $L_{s}[\dot{x}(t)]$ and $L_{s}^{\prime}\left[\dot{x}^{\prime}(t)\right]$ are of the same form, and that $L_{n}[\dot{x}(t), x(t), t]$ and $L_{n}^{\prime}\left[\dot{x}^{\prime}(t), x^{\prime}(t), t\right]$ have exactly the same form. 


\section{Definition of forces}

The energy function $\mathcal{E}$ in $\mathrm{CM}[11,12]$ is given by

$$
\mathcal{E}=\dot{x} \frac{\partial L}{\partial \dot{x}}-L
$$

where $L$ is a Lagrangian. If $L=L_{s}[\dot{x}(t)]$, then $\mathcal{E}=E_{\text {tot }}=$ const, with $E_{\text {tot }}$ being the total energy; in the case considered here, $E_{t o t}=E_{k i n}=c_{o} \dot{x}^{2} / 2$. However, when $L[\dot{x}(t), x(t), t]=$ $L_{s}[\dot{x}(t)]+L_{n}[\dot{x}(t), x(t), t]$, the energy function then becomes

$$
\mathcal{E}=\frac{1}{2} c_{o} \dot{x}^{2}(t)+\frac{1}{2} \dot{f}_{1}(t) x^{2}(t)-\frac{1}{2} v_{o}\left[f_{1}(t)+\dot{f}_{1}(t) t\right] x(t)-\left[\dot{f}_{6}(t) t+f_{6}(t)\right] .
$$

Since the first term on the RHS is equal to $L_{s}[\dot{x}(t)]$, we denote the remaining terms as $L_{r}[\dot{x}(t), x(t), t]$ and define $L_{\mathcal{E}}[\dot{x}(t), x(t), t]=L_{s}[\dot{x}(t)]+L_{r}[\dot{x}(t), x(t), t]$. In general, the Lagrangian $L_{r}[\dot{x}(t), x(t), t]$ is not the null Lagrangian but it may be converted to one if, and only if, $f_{1}(t)$ is of a special form. To determine this form, we substitute $L_{r}[\dot{x}(t), x(t), t]$ into the Euler-Lagrange equation and find $f_{1}(t)=C_{r} \exp \left[v_{o} / 2\left[\left(u_{o}-v_{o} / 2\right) t+x_{o}\right]\right.$; because of its special for, we keep $f_{1}(t)$ to be arbitrary.

Substituting $L_{\mathcal{E}}[\dot{x}(t), x(t), t]$ into the Euler-Lagrange equation, the following equation of motion is obtained

$$
\ddot{x}(t)=F[x(t), t],
$$

where

$$
F[x(t), t]=\frac{1}{c_{o}} \dot{f}_{1}(t) x(t)-\frac{v_{o}}{2 c_{o}}\left[f_{1}(t)+\dot{f}_{1}(t) t\right] .
$$

This shows that the derived exact null Lagrangian can be used to introduce the force $F[x(t), t]$ into the law of inertia and convert it into the second Newton law of dynamics. The resulting force is linear in the dependent variable and can be of any dependence on the independent variable since $f_{1}(t)$ is an arbitrary function of $t$ as long as it remains continuous and differentiable. Note that $x(t) \neq u_{o} t+x_{o}$ as the solution of the inhomogeneous equation of motion is now different and depends on the form of $F[x(t), t]$.

Now, if $F[x(t), t]$ does not depend on $x(t)$, then the force $F(t)$ can be any function of time as long as it is continuous and differentiable, with the additional requirement that $f_{1}(0)$ satisfies Eq. (13); the function $f_{1}\left(t_{e}\right)$ is not restricted. All constants in this force are uniquely determined by the initial conditions. Because of the dependence of $F(t)$ on the dependent variable $x(t)$, the derived inhomogeneous equation of motion is not Galilean invariant; similarly, the Lagrangian $L_{\mathcal{E}}[\dot{x}(t), x(t), t]$ used to obtain this equation is also not Galilean invariant. In order to make them Galilean invariant, it is required to repeat the procedure described in Section 3 and 4 of this Letter, which will not be done here.

The main advantage of $f_{1}(t)$ being an arbitrary function of time is that a time-dependent force that drives a dynamical system may be represented by this function. In general, the exactness imposed on the gauge functions sets up the initial value of the force, or in other words, its amplitude (at $t=0$ ) through the given initial conditions. This shows that not all time-dependent forces may satisfy these conditions. This is the physical limitation on the 
force caused by the exactness. The effect of the exactness is even more prominently seen in a special case of $f_{1}(t)=c_{1}=$ const [6]; in this case, the resulting force is constant and uniquely determined by the initial conditions.

Our method of defining $F(t)$ allows us to convert the law of inertia into the second Newton law of dynamics. Our results show that this conversion requires the Lagrangian formalism and Galilean invariance, and that it does not guarantee that the resulting inhomogeneous equation of motion remains Galilean invariant; however, if the Galilean invariance is imposed on $F(t)$ then its form is further constrained.

Finally, let us point out that our novel approach and obtained results allow us to relate the first and second laws of dynamics, and solve the problem of the circular definition of the inertial mass and forces in Newtonian mechanics [1,2].

Acknowledgments We are grateful to an anonymous referee for carefully reading our manuscript and providing many detailed comments and suggestions that helped us to significantly improved the revised version of this paper. We also thank L. Vestel for checking our derivations and comments on the manuscript.

\section{References}

[1] M. Jammer, Concepts of Mass in Contemporary Physics and Philosophy, Princeton University Press, Princeton, NJ, 2000

[2] M. Jammer, Concepts of Mass in Classical and Modern Physics, Dover Publications, Inc., Mineola, NY, 1997

[3] L.D. Landau and E.M. Lifschitz, Mechanics, Pergamon Press, Oxford, 1969

[4] V.I. Arnold, Mathematical Methods of Classical Mechanics (Springer, New York, 1978)

[5] J.-M. Levy-Leblond, Comm. Math. Phys. 12 (1969) 64

[6] Z.E. Musielak and T.B. Watson, Phys. Let. A, 384 (2020) 126642

[7] Z.E. Musielak, J. Appl. Math., under review (2020)

[8] M. Crampin and D.J. Saunders, Diff. Geom. and its Appl., 22 (2005) 131

[9] D.R. Grigore, J. Phys. A 28 (1995) 2921

[10] D. Krupka and J. Musilova, Diff. Geom. and its Appl. 9 (1998) 225

[11] H. Goldstein, C.P. Poole, J.L. Safko, Classical Mechanics (3rd Edition) Addison-Wesley, San Francisco, CA, 2002

[12] J.V. José, E.J. Saletan, Classical Dynamics, A Contemporary Approach, Cambridge Univ. Press, Cambridge, 2002 